George Devereux zum 75. Geburtstag 


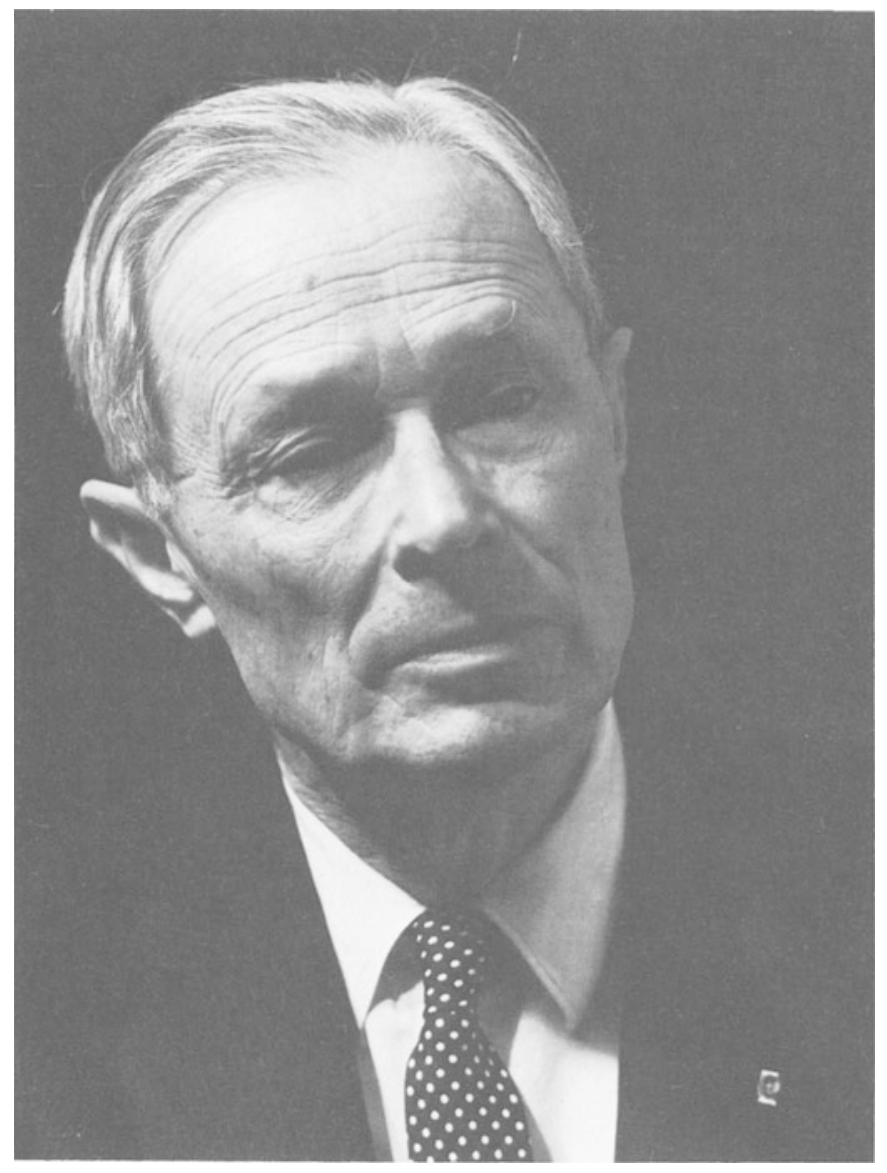

George Devereux 


\title{
George Devereux zum 75. Geburtstag. Eine Festschrift
}

\author{
Herausgegeben im Auftrag der \\ Arbeitsgemeinschaft Ethnomedizin \\ von \\ Ekkehard Schröder und Dieter H. Frießem
}

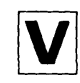

Springer Fachmedien Wiesbaden $\mathrm{GmbH}$ 
Erläuterungen zu den Abbildungen der Umschlagseite stehen auf Seite 32.

Das Frontispiz von George Devereux stammt aus dem Jahre 1972.

(C) Springer Fachmedien Wiesbaden 1984

Ursprünglich erschienen bei Friedr. Vieweg \& Sohn Verlagsgesellschaft mbH, Braunschweig 1984.

Die Vervielfältigung und Übertragung einzelner Textabschnitte, Zeichnungen oder Bilder, auch für Zwecke der Unterrichtsgestaltung, gestattet das Urheberrecht nur, wenn sie mit dem Verlag vorher vereinbart wurden. Im Einzelfall muß über die Zahlung einer Gebühr für die Nutzung fremden geistigen Eigentums entschieden werden. Das gilt für die Vervielfältigung durch alle Verfahren einschließlich Speicherung und jede Übertragung auf Papier, Transparente, Filme, Bänder, Platten und andere Medien.

ISBN 978-3-528-07920-8

ISBN 978-3-663-19640-2 (eBook)

DOI 10.1007/978-3-663-19640-2 


\section{Inhalt}

\section{Vorwort}

"Ich habe das Recht, ich zu sein und kein anderer".

Ein Gespräch zwischen Georg Devereux und Ekkehard Schröder, geführt am 31. Januar 1984 in Antony

\section{Normal und anormal}

Dieter H. Frießem: Devereux und die Psychiatrie. Gedanken beim Wiederlesen von "Angst und Methode"

Wolfgang Blankenburg: Ethnopsychiatrie im Inland.

Norm-Probleme im Hinblick auf die Kultur- und Subkulturbezogenheit psychiatrischer Patienten

Werner F. Bonin: Über Wunderlinge, Sonderlinge, Käuze zu ihrer Funktion in der Gemeinschaft und zur Konnotation der Begriffe

Michael Knoll: Extrem normal - Paradoxie und Hoffnung einer psychiatrischen Freiheitsethologie

Gunter Hofer: Besessenheit, ein Phänomen der menschlichen Lebenswelt

\section{Psychiatrie und Kultur}

Emil Zimmermann: Krankheit und Kranksein aus soziokultureller Sicht. Ein Beitrag zur Medizin der Migration

András Kelemen: Die Hauptfaktoren der Psychohygiene in der traditionellen türkischen Gesellschaft

Bruno G. Claver: Relations familiales et demandes thérapeutiques en Côte d'Ivoire / Familienstruktur und Therapiebedarf in der Elfenbeinküste 


\section{Ethnomedizin}

„Einen Lebensbaum für Professor Devereux". Kurzbeschreibung einer Geburtsvorbereitung im Dschungel von Hans Kalipke

Hans Kalipke: Schwangerschaft und Geburt bei den Sakai auf Sumatra

Norbert Kohnen: Traditionelle Gebräuche um Schwangerschaft und Geburt auf den Philippinen

Winfried Effe/sberg: Krid, ein kulturgebundenes Syndrom von den Kei-Inseln

Wolfgang Bichmann: Die Rolle der traditionellen Medizin in der Entwicklung Afrikas

Barbara Mainzer-Heyers: Die traditionellen Krankheiten und ihre Therapie in San Pedro de Moya/Huancavelica

\section{Freie Beiträge}

Thomas Hauschild: Abwehrmagie und Geschlechtssymbolik im Mittelmeerraum

Michel Erlich: Infibulation feminine et phallicisation de la vulve / Infibulation und Phallizisierung der Vulva

Alfred Dieck: Zur Heilkunde der Germanen - Materialien aus

Edda und Sagas

Eno Beuchelt: Die Fernreise als Initiation

Béla Gunda: Heilpflanzen in einem ungarischen Dorf der

Karpaten-Ukraine

Hartmut Zinser: Freuds Konstruktionen gesellschaftlicher Synthesis

Ulrich Sonnemann: Bilder der Wissenschaft. Sieben Geschichten

aus einem Satz für George Devereux

Klaus E. Müller: Ursprung, Sein und Ewigkeit: der Dualismus als zentrales Problem der menschlichen Existentialität

Wolfdietrich Schmied-Kowarzik: Karl Marx und die philosophische Grundlegung einer geschichtsmaterialistischen Kulturtheorie

\section{Anhang 1}

Autoren und Mitarbeiter dieser Festschrift

Dieter H. Frießem: Vorläufige Bibliographie der Schriften von George Devereux

Anhang 2

Die Mitarbeiter dieser Festschrift 


\section{Vorwort}

Am dreizehnten September 1983 feierte George Devereux seinen 75. Geburtstag. Die Idee, ihn zu diesem Anlaß mit einer Festschrift zu ehren, entsprang dem regen Kontakt zwischen dem Jubilar und jenen Mitgliedern unserer Arbeitsgemeinschaft, denen er als "Georg" verständnisvoller Freund und kritischer Diskussionspartner zu bleiben angeboten hatte.

Im Kreise dieser Arbeitsgemeinschaft sprach George Devereux erstmals im Mai 1977 in Heidelberg auf deren dritter internationaler Fachtagung und warnte mit seinem damaligen Referate über das, was er "die Verunsicherung der Geisteskranken“ nannte, im Rahmen des Gesamtthemas „Familienkonzepte in ihrer Bedeutung als Elemente für die soziale Sicherung" vor Idealisierungen fremdethnischer Sachverhalte. In seinen dortigen Diskussionsbeiträgen klangen spontan auch Leitmotive seines bewegten Lebens als Ethnologe, klinischer und freier Psychoanalytiker und Altertumswissenschaftler an, die im ersten Beitrag dieser Sammlung weiter verdeutlich werden.

Ausgehend von den "exakten", nomothetischen Wissenschaften der Mathematik und Physik am Anfang seines Studiums ist George Devereux schließlich zum Vater der Methodik der Ethnopsychoanalyse geworden, einer Arbeitsrichtung, welche generalisierende und individualisierende Verfahren i.S. Rickerts, Methodenkritik und Praxis gleichermaßen miteinander vereint. "Angst und Methode", sein mittlerweile in fünf Sprachen erschienenes, bereits während seiner Lehrjahre als Feldforscher konzipiertes Hauptwerk, hat erst spät seine Wirkung zu entfalten begonnen, ein rezeptions- und wirkungsgeschichtliches Unikum, allenfalls vergleichbar mit den Wegen und Umwegen, die Elias' „Prozeß der Zivilisation“ genommen hat. Indessen ist George Devereux, an Interessen seiner von den besten Traditionen europäischen Bildungsbürgertums geprägten Jugendjahre anknüpfend, längst auf dem Feld der Gräzistik tätig geworden und hat hier schon wieder mehrere Monographien und eine Vielzahl von Untersuchungen vorgelegt. Im Zusammenhang mit Baubo, jener Figur der altorphischen Demetersage, welcher er eine weit ausholende Einzeldarstellung gewidmet hat, stehen auch die Titelvignetten dieser Festschrift.

George Devereux ist bis heute ein unermüdlicher Arbeiter geblieben, und dies trotz seiner angegriffenen Gesundheit. Auf die Auswertung und Veröf- 
fentlichung seiner nunmehr fünfzig Jahre zurückliegenden Feldaufzeichnungen bei den Sedang sind wir alle gespannt.

Die Herausgeber dieser Sammlung wünschen George Devereux im Namen der Arbeitsgemeinschaft Ethnomedizin und all jener, die - zum Teil als seine persönlichen Freunde - zu deren Gelingen beigetragen haben, noch viel Elan, wissenschaftliche Neugierde und Anregung, den Lesern aber Entdekkerfreuden.

Ekkehard Schröder

Saarbrücken und Stuttgart,

Dieter H. Frießem im Juni 1984 